\title{
THE EFFECT OF THE COVID-19 PANDEMIC ON BREAST CANCER STAGES
}

\author{
Andrei Alves de Queiroz¹, Ana Maria Kemp1, Vanessa Ribeiro Lopes¹, Debora Garcia y Narvaiza1 \\ ${ }^{1}$ Hospital de Clínicas Luzia de Pinho Melo - Mogi das Cruzes (SP), Brazil.
}

Introduction: The COVID-19 pandemic brought us several behavioral changes, including social isolation, which impacted breast cancer screening around the world, including in Brazil. With a decrease in the number of exams performed, the risk of breast cancer detection in later stages increases, negatively impacting the prognosis of the disease. Objectives: To analyze and compare breast cancer stages in patients at a hospital in Mogi das Cruzes - SP before and during the pandemic. Methods: Retrospective, observational, and analytical study carried out through analysis of records of patients admitted to the outpatient clinic between January 1, 2019 and December 31, 2020. This service is reference for 11 municipalities covering an estimated population of more than three million inhabitants. For statistical analysis, and to allow cases referred in the pre-pandemic period to be considered as such, the pandemic period was determined to be from April 1, 2020 on. Results: A total of 331 breast cancer patients who started treatment between 2019 and 2020 were identified. 23.6\% fewer cases were admitted during the pandemic, with 102 patients attended, an average of 11.3 patients/month, while before the pandemic the average was 15.3 patients/month. There was a statistically significant difference of four patients/month $(\mathrm{p}<0.001)$. The mean age of the patients also varied significantly, the patients being younger during the pandemic, with a difference of 3.5 years ( 57.8 vs $54.3 ; \mathrm{p}=0.03$ ). In order to analyze the effect of the pandemic in breast cancer stages, the number of cases was studied each month, and a significant drop was identified in the stages 0 and I (3.2 vs 0.7 cases/month; $\mathrm{p}<0.001$ ), with no increase identified in the other stages. A multivariate analysis also identified a significant drop in diagnoses at early stages, even after considering confounding factors (age), with OR=0.29 (95\%CI 0.11-0.63; $\mathrm{p}=0.004)$. No significant change was identified in cases of locally advanced or metastatic disease (stages III and IV), with an OR of 0.86 (95\%CI 0.51-1.41; $\mathrm{p}=0.55)$. It is important to remember that these early-stage patients are often asymptomatic and diagnosed, mostly, through screening tests. Conclusions: The deficit in patients diagnosed at early stages was not accompanied by an increase in advanced stages. It is possible that there are still many women with undiagnosed breast cancer, but with the possibility of early identification. 